\section{$\underset{\substack{\text { hommes } \\ \text { \& migrations }}}{ }$}

\section{Hommes \& migrations}

Revue française de référence sur les dynamiques

migratoires

$1314 \mid 2016$

Migrations chinoises et générations

\title{
«C'est écrit sur ton visage »
}

La construction identitaire des Chinois de la deuxième génération en Hongrie dans un contexte de " nationalisme à distance »

\section{Fanni Beck}

Traducteur : Catherine Guilyardi

\section{(2) OpenEdition}

\section{Journals}

Édition électronique

URL : http://journals.openedition.org/hommesmigrations/3638

DOI : 10.4000/hommesmigrations.3638

ISSN : 2262-3353

Éditeur

Musée national de l'histoire de l'immigration

Édition imprimée

Date de publication : 1 avril 2016

Pagination : 63-69

ISBN : 978-2-919040-35-3

ISSN : 1142-852X

Référence électronique

Fanni Beck, « «C'est écrit sur ton visage » », Hommes \& migrations [En ligne], 1314| 2016, mis en ligne

le 01 avril 2019, consulté le 20 avril 2019. URL : http://journals.openedition.org/

hommesmigrations/3638; DOI : 10.4000/hommesmigrations.3638 


\title{
"C'EST ÉCRIT SUR TON VISAGE" LA CONSTRUCTION IDENTITAIRE DES CHINOIS DE LA DEUXIĖME GÉNÉRATION EN HONGRIE DANS UN CONTEXTE DE "NATIONALISME À DISTANCE"
}

par FANNI BECK, doctorante en anthropologie, Eötvös Loránd University, Social Sciences Department.

\author{
Les descendants de Chinois nés à l'étranger, comme \\ ici en Hongrie, représentent un défi idéologique et culturel \\ pour les autorités de la République populaire de Chine \\ qui tentent d'éveiller chez eux l'attachement pour le pays \\ de leurs parents. Camps de vacances, concours de beauté, \\ les initiatives se multiplient pour valoriser leurs racines \\ chinoises. Cette façon de nourrir à distance le nationalisme \\ de cette jeunesse transnationale se heurte à des identités \\ hybrides qui se construisent entre plusieurs territoires où elles \\ ont à négocier leur reconnaissance et leur place.
}

L'existence d'une deuxième génération de migrants soulève singulièrement la question de notre compréhension de l'ethnicité et de l'appartenance nationale. Qui sont-ils ? D'où sont-ils ? La complexité qui sous-tend ces questions apparemment banales nous oblige à croiser deux domaines de recherches sur les questions transnationales ${ }^{1}$ : l'un se concentre sur l'aspect nationaliste du transnationalisme, c'est-à-dire le "nationalisme à distance ${ }^{2 "}$, l'autre observe la deuxième génération de migrants à travers le prisme du transnationalisme, c'est-à-dire ceux élevés dans un champ social transnational ${ }^{3}$. Tout en analysant les constructions identitaires des Chinois de la deuxième génération en Hongrie, le concept d'hybridité proposé par Ien Ang permet de comprendre la présence simultanée d'émotions opposées. En juxtaposant le concept d'hybridité à celui de nationalisme à distance ${ }^{4}$, il s'agit de montrer que la construction d'une identité hybride pour la deuxième génération crée un terrain fertile pour les pratiques de construction nationale extraterritoriale.

Cette hypothèse est développée grâce à l'analyse de deux initiatives de la République populaire de Chine (RPC), dont le but est d'éveiller le sentiment nationaliste chez les descendants de Chinois, nés hors 
de Chine : les colonies de vacances gouvernementales de retour aux racines, "Root-seeking camps", réservées aux jeunes d'outre-mer, et le concours de beauté "Miss Chinoise Europe". Les constructions identitaires hybrides de la deuxième génération sont, selon cette étude, la Avant l'apparition cible parfaite de ces deux prade la deuxième - pour ne pas dire la dernière - génération de migrants, il était évident que la conscience nationale découlait naturellement de l'héritage racial chinois. tiques de construction nationale extraterritoriale. Ces pratiques surfent sur un discours racialisé de l'ethnicité qui coïncide avec l'expérience d'exclusion raciale que vit la deuxième génération de Chinois en Hongrie. Par là, elles contribuent à forger ces identités contestées, mais, nous le verrons, pas de la façon dont elles visent à le faire. Deux entretiens seront mobilisés pour illustrer comment des concepts théoriques et des politiques nationalistes opèrent au niveau de l'individu 5 . D'abord, il est nécessaire de présenter les acteurs de cette construction nationale extraterritoriale : premièrement, la RPC et sa politique d'émigration, deuxièmement, la seconde génération de migrants et son identité hybride.

\section{Construire la nation de l'étranger: une politique de l'État chinois}

Le transnationalisme est souvent perçu comme un affaiblissement de l'État-nation. Cette idée demande à être discutée car elle ne prend en compte que le pays d'accueil, oubliant que le pays d'origine est également un État-nation. Or le pays d'origine - bien que faiblissant - trouve une nouvelle opportunité de construire sa nation de façon extraterritoriale. Le terme de "construction à distance de la nation" a été entériné et utilisé de fait par les chercheurs du transnationalisme $e^{6}$. La RPC est connue pour sa politique particulièrement favorable à l'émigration : depuis 1978, quitter le pays est vu comme un acte moderne et patriotique et est encouragé de façon institutionnelle et dans les discours ${ }^{7}$. L'image du migrant est celle d'un courageux constructeur de ponts aidant la nation à entrer dans le monde moderne. Reconnaissant l'intérêt économique de garder de bonnes relations avec ses expatriés, le gouvernement chinois participe de façon active à la création d'une communauté transnationale dans la diaspora chinoise.

Le nationalisme à distance de la RPC repose sur l'idée de liens de sang et d'héritage ${ }^{8}$. Avant l'apparition de la deuxième - pour ne pas dire la dernière - génération de migrants, il était évident que la conscience nationale découlait naturellement de l'héritage racial chinois. Ce discours était totalement en accord avec le désir des migrants de la première génération : exclus du fait d'être racialement distincts comme membres marginaux de la société d'accueil, ils désiraient plus que tout appartenir à une communauté supérieure, être honorés par une mère-nation aimante. Utiliser des slogans nationalistes traditionnels qui réveillent le sentiment de nostalgie fonctionne bien avec ceux qui ont des souvenirs de la mère-nation, mais pas avec ceux qui ne sont jamais sortis de leur village hongrois. La présence de la deuxième génération met ainsi au défi le discours nationaliste traditionnel' ${ }^{9}$.

\section{L'hybridité : un état de suspension constante dans un entre-deux}

Si les personnes visées par les politiques de construction nationale à distance dont nous parlons ici sont nées et élevées en dehors de la Chine, 
même personne peut se définir comme chinoise, européenne, hongroise ou hybride, selon le point de référence contre lequel elle construit son identité du moment. Ce qui est constant dans ces discours est le clivage entre l'aspect physique (Chinois) et la façon dont ils pensent (Hongrois, ou plus largement Européen). Ce qui les met dans une situation décrite par Saïd comme un "état généralisé de sans-domicile-fixe ${ }^{11}$ ” illustré par un de nos interlocuteurs déclarant "ne pas avoir sa propre nationalité". L'état de suspension constante dans un entre-deux crée une cible idéale pour les initiatives nationalistes.

\section{La demande répond à l'offre}

Selon Andrea Louie ${ }^{12}$, la RPC pense que la loyauté chinoise vis-à-vis de la terre natale, et donc de la nation chinoise, est incarnée par les Chinois d'outre-mer dans leur héri-

Les passants, rue de Belleville, mai 2016. @ Camille Millerand

leur aspect physique a indéniablement quelque chose de "chinois". Pour interpréter l'ambiguïté entre l'apparence extérieure et l'identité intérieure, le concept d'hybridité est un outil précieux d'analyse. Il n'est pas une solution, mais un outil heuristique pour analyser cet imbroglio. Il permet de traiter de façon simultanée les désirs contradictoires de revendiquer sa différence et de se sentir appartenir à un tout. Il ne s'agit pas de chercher à réconcilier ou séparer ces sentiments, mais de reconnaitre l'impossibilité de résoudre les contradictions ${ }^{10}$.

Une identité se forme toujours par opposition à une autre. Elle n'est jamais stabilisée ou fixe. Une tage racial. La "sinité" est conçue comme une essence raciale qui connecte les personnes à la nation chinoise par le sang, quel que soit l'endroit du globe où elles vivent ou sont nées. Nous pensons au contraire qu'en dépit des postulats de la RPC et de la culture collective chinoise, le sentiment national ne découle pas naturellement d'un héritage racial ${ }^{13}$, mais du processus qui confronte l'individu avec cet héritage de façon répétée. La nécessité de se trouver des racines amène la RPC à en fournir. La citation de Nam, un jeune homme né en 1992 à Santiago du Chili, habitant Budapest au moment de l'entrevue, illustre l'efficacité de ces méthodes de la Chine sur des gens qui rencontrent des difficultés d'identification : " $\mathrm{La}$ culture chinoise est vraiment forte, comme je l'ai dit, et, je dirais que quand tu es né de cette façon tu as deux choix. Le premier, c'est accepter que tu es chinois, 
et alors tu te sens y appartenir pour toujours. L'autre est de rejeter le fait que tu es chinois, et je ne le recommanderais pas, parce que c'est écrit sur ton visage. C'est trop fort, tu ne pourras jamais te couper de la culture chinoise."

\section{À la recherche de racines : les colonies de vacances pour les Chinois de la diaspora}

Les colonies de vacances "À la recherche de racines" (Root-seeking camps), réservées aux Chinois de l'étranger, sont un bon exemple de croisement entre la difficile construction d'une identité hybride et les politiques de construction nationale extraterritoriale. Les publications officielles sur ces colonies ${ }^{14}$ listent clairement les effets voulus et les buts recherchés au travers de cette initiation : si les candidats pouvaient connaître leur patrie d'origine, ils l'adoreraient. "Les membres de la jeune génération de la diaspora chinoise ont une compréhension réduite de la culture tradition-

La contribution des capitaux des Chinois de la diaspora dans

cette transformation

est mise en valeur avec

la claire intention d'encourager

les futurs investissements

présentés comme un acte

patriotique. nelle de leurs ancêtres, sans parler de l'inexistence d'un sentiment national. Nous avons donc basé ces colonies de vacances sur la culture nationale chinoise $e^{15}$." Le nom générique de ces colonies est "Zhongguo xun gen zhi lu-Voyage à la recherche de ses racines chinoises ${ }^{16 "}$.

Mais les candidats sont rarement intéressés par leurs "racines" et veulent juste s'amuser. Les récits officiels montrent de façon récurrente une Chine en pleine transformation en un pays moderne et international. La contribution des capitaux des Chinois de la diaspora dans cette transformation est mise en valeur avec la claire intention d'encourager les futurs investissements présentés comme un acte patriotique ${ }^{17}$. C'est une approche donnant-donnant : “Avec l'intégration progressive de la Chine dans le jeu mondial et son immense marché potentiel, de plus en plus de personnes étudient le chinois pour améliorer leur perspective d'emploi", écrit Shan Chun, un expert de l'Académie chinoise des sciences sociales, dans une campagne de promotion sur le site du China Daily ${ }^{18}$.

Cette approche trouve un écho chez les jeunes Chinois de la diaspora qui voient dans le fait de pouvoir faire des affaires en - ou avec - la Chine le principal - sinon le seul - intérêt d'être Chinois. Intégrant l'attitude individualiste de jeunes élevés en Occident, les raisons de leur motivation ne sont pas d'agir pour le bien du pays d'origine mais pour leur propre bien. Comme le montrent ces paroles de Nan, ce discours fonctionne car il résonne avec l'expérience de discrimination sur le marché local de l'emploi : "Le plus dur, c'est de trouver un travail. Si je postule et qu'il y a quelqu'un en compétition avec moi, si on est exactement pareil, ils le prendront lui, juste (...) parce qu'ils supposent qu'ils partagent la même culture."

Une chose que les concepteurs de ces programmes n'ont pas pris en compte est le choc culturel que subissent les Chinois de la diaspora la première fois qu'ils viennent en Chine, comme le dit Nan : "La culture chinoise est assez forte, si je peux le dire ainsi. Pour la plupart des étrangers, moi y compris, venir en Chine représente un choc. D'être simplement dans la rue et de voir beaucoup de gens, une mer de chevelures noires, c'est assez surprenant."

Le jeune homme se considère comme un "étranger", de toute évidence, et il a subi le même choc culturel que tous les étrangers qui viennent en Chine. Mais son expérience diffère car il s'attendait à arriver chez lui. Or il s'est senti plus étranger que jamais. "C'est un point crucial pour me définir. Depuis le tout début, depuis que je suis entré en classe primaire, j’ai toujours été diffèrent des autres, parce que j'étais chinois et, à la fois, je ne l'étais pas. Je ne me suis jamais senti à $100 \%$ Chilien alors que j’ai

14. Ibid. 15. Publicités du Go Chengdu pour des colonies de vacances pour Chinois de l'étranger : www.gochengdu.cn/news/ culture-tourism/young-overseas-chinese-attend-root-seeking-summer-camp-in-chengdu-a618.html\#sthash.eJPgftWc.dpuf 16. Voir le site official : http://summercamp.hwjyw.com/ 17. Voir Pál Nyíri, op. cit.; Andrea Louie, "Re-territorializing transnationalism: Chinese Americans and the Chinese abroad", op. cit.; "Crafting places through mobility: Chinese American 'roots-searching' in China", op. cit. 18. www.china.org.cn/english/16706.htm 
grandi là-bas. Et puis je suis allé en Chine : je ressemble à un Chinois, je parle le chinois mais les Chinois me traitaient comme un étranger. J'ai senti que je n'étais pas chinois non plus. La plupart des gens qui sont étrangers, moitié Chinois comme moi, sont de nulle part. Nous sommes un mélange de nos pays et de la Chine."

Si on en croit ces mots, la colonie de vacances, même si elle a atteint son but premier, a un effet contreproductif. À regarder de près, on remarque qu'il y a un impact non intentionnel : l'usage du "nous" souligne la création d'un sens d'appartenance à une communauté de personnes qui sont de "nulle part", une communauté transnationale qui partage l'héritage implacable des caractéristiques physiques chinoises. Même si c'est de manière indirecte, l'initiation a donc atteint son but car elle permet de renforcer les liens des jeunes avec la Chine.

\section{Les concours de beauté Miss Chine-Europe}

Des concours de beauté chinois sont organisés sur toute la planète, de Montréal aux Philippines. Ils ne sont pas organisés directement par le gouvernement de la RPC mais participent de façon importante à la construction nationale à distance, car ils facilitent l'expression de l'identité chinoise des migrants de la deuxième génération. Les candidates doivent montrer qu'elles sont "chinoises" et "européennes" en même temps. Cette situation est un parfait cas d'hybridité : les identités parallèles et disputées doivent être mises en scène simultanément. 
La nature de l'événement place le corps racisé au centre de l'attention. Le corps - et parfois seul le corps - reste chinois pour quasiment tous les descendants de Chinois $^{19}$. Comme dans le cas des colonies de vacances, il paraît évident que les candidates doivent avoir certaines caractéristiques physiques ethniques chinoises. Mettre le corps des jeunes femmes d'origine chinoise sous les projecteurs, alors qu'il est la principale source de leur exclusion et de leur marginalisation, est paradoxal à bien des égards. Comme le souligne Yiu Fai Chow dans son étude sur "Miss China Europe", "l'hyper-visibilité de la femme chinoise de la diaspora lors de lévènement (...) contraste douloureusement avec leur invisibilité quotidienne, que ce soit aux Pays-Bas, en Chine ou ailleurs dans le monde $e^{20}$. Ces concours de beauté peuvent être vus comme un acte d'“auto-orientalisation ${ }^{21 " . ~ E n ~}$ célébrant le corps racisé, la narration qui l'entoure change, présentant le corps non plus comme "différent" mais "exotique". Ce discours a été adopté par une des participantes, Miss Amitié 2014 (Sissy, née en 1995, Budapest) : “Arrivée au lycée, j’avais compris que jétais exotique. Quand j'allais dans un magasin, ou dans la rue, quand je parlais à quelqu'un qui me disait: 'Comme vous êtes belle, comme vous parlez bien hongrois.' Je répondais toujours: 'Bien sûr, je suis née ici.' J'ai reçu plein de retours positifs du monde extérieur grâce à mon visage asiatique exotique, j’ai pu l'utiliser comme quelque chose de positif, de très spécial."

Ce concours permet aux candidates de répondre au besoin d'exprimer leur identité. Sissy dit encore : "Il y avait une question sur pourquoi je pensais pouvoir être Miss Chine Europe. Je me suis mise à réfléchir à ce qui, à part mon visage chinois, faisait de moi une Chinoise. Cela n'a pas été facile, parce que je n'y avais jamais réfléchi avant." Cette expérience lui a servi de catalyseur pour se forger une identité chinoise, ce qui ne lui avait pas semblé nécessaire avant. Elle a trouvé plus difficile de s'identifier à une Chinoise qu'à une Européenne : “Je n'ai pas besoin d'être européenne parce que je pense à $99 \%$ comme les Européens. Ce que je dois chercher, c'est le $1 \%$ de chinois en moi, mais je ne l'ai toujours pas trouvé. Je n'ai pas besoin d'être européenne, je le suis déjà. Si je vais en Chine, ils ne me verront pas comme une Chinoise." Le fossé entre ce à quoi elle ressemble - une Chinoise - et sa façon de penser - européenne - est évident. Quand elle essaye de définir son identité, une partie est choisie volontairement, l'autre a besoin d'être gérée. La contradiction semble insoluble. Son "européanité" devient évidente en contraste avec sa "sinité" qui a besoin d'être mise en valeur. Selon l'officiel TVB, en charge des concours de beauté Miss Chinese International, l'intention originelle de ces concours est de "permettre [aux Chinoises d'ailleurs] de se sentir plus proches de la culture chinoise". Mais, en réalité, les candidates sont confrontées à leur illégitimité culturelle, souvent moquées pour leur incompétence linguistique. Au lieu de les faire se sentir "plus proches", cette expérience leur fait vivre un nouveau sentiment de marginalité. "Le fait d'être perçues comme des Chinoises puis d'être ensuite marginalisées parce que pas assez Chinoises [parce qu'elles ne remplissent pas toutes les attentes culturalistes] crée les mêmes sentiments d'impuissance et d'inégalité que ceux que ressent la diaspora chinoise dans sa vie quotidienne ${ }^{22}$." La présence d'étudiantes chinoises, venues en Europe pour leurs études supérieures, mais nées et élevées en RPC, parmi les candidates, montre que les critères ne sont pas très clairs : aux yeux des spectateurs et des candidats, qui les appellent "vraies Chinoises" ou "Chinoises typiques", elles ne sont pas assez européennes pour devenir Miss Chine-Europe. Leur présence a poussé la majorité des candidates, nées et élevées en Europe, à exprimer leurs différences et à se définir comme des Européennes.

\section{Des identités sur le podium}

La langue est un marqueur important de l'autoidentification des migrants. Que cela peut-il signifier d'être un(e) "vrai(e)" Chinois(e) ? La plupart des 
gens qui gardent les caractéristiques physiques de leur "sinité" pensent qu'ils ont l'obligation, que c'est de leur responsabilité, de maîtriser le chinois (bien qu'il ne soit pas évident dans la plupart des cas de savoir si l'on parle du mandarin ou d'un autre dialecte). La citation qui suit montre comment "l'hégémonie de la sinité visuelle ${ }^{23 "}$ est vue comme une évidence dans la vie courante : "Ici, chez moi, si vous ne parlez pas hongrois ou anglais, les gens comprennent parce que je ressemble à une Chinoise, par contre si je ne parle pas chinois, alors que je ressemble à une Chinoise, les gens me regardent bizarrement."

Cette citation montre aussi comment le discours sur les attentes envers le corps racisé devient une évidence pour tous les acteurs concernés. La majorité des pays de résidence, comme l'opinion publique et officielle en Chine, demandent aux individus quelque chose qui n'est pas du tout évident. Le pouvoir de pénétration d'un discours qui lie apparence visuelle et maîtrise de la langue est une expérience vécue par tous ceux qui vivent avec un visage chinois à l'étranger, encore plus par ceux qui ne parlent pas la langue. Pourtant, parler chinois ne fait pas automatiquement de vous une Chinoise. Une candidate raconte comment elle s'est sentie, au milieu de "Chinoises typiques", et décrit la pression qu'elle a vécue lors du concours pour "prétendre être chinoise". Elle a eu l'impression de devoir rejeter son identité si elle voulait gagner : "J'étais qui j'étais, cela m'allait bien... J'ai toujours pensé que jétais quelqu'un de valeur et je le pense toujours, j’ai réussi tellement de choses grâce à ça, d'être si... spéciale dans un sens. Donc jétais moi-même et je ne prétendais pas être quelqu'un d'autre. Mais cette fois, cela ne m'a menée nulle part. J'aurais dû faire semblant d'être chinoise, jouer le jeu. Si j'avais pu jouer le jeu, mais je n'ai pas pu, je ne peux pas me comporter comme une Chinoise..."

Bien qu'elle se prépare à une carrière d'actrice à Londres, jouer le rôle d'une Chinoise dépassait ses compétences. Penser comme le font les Chinois est en partie impossible et en partie quelque chose à éviter afin de préserver son identité. Penser et agir comme une Chinoise était comme abandonner sa personnalité. Contrairement aux attentes des organisateurs, le concours a eu pour résultat de renforcer chez la candidate interrogée son sentiment d'européanité qui la distinguait des autres Chinoises "typiques". Toutefois, le concours de beauté a eu pour effet de lui faire prendre conscience quêtre chinoise n'était pas qu'une question d'apparence. Cela l'a décidée à s'investir davantage dans l'étude de la culture chinoise. "Avant je pouvais lire le chinois, après cela jétais encore plus intéressée. Tant qu’à être chinoise, autant connaître plus de choses sur la Chine."

\section{Conclusion}

Les initiatives décrites dans cet article confrontent intentionnellement les participants à la complexité de leur identité nationale et ethnique, et les poussent à délimiter leurs parts hongroise/ européenne/occidentale de leur part chinoise. Elles peuvent être considérées comme des outils pour la construction nationale à distance, car elles reflètent la volonté de la RPC de maintenir des relations avec les migrants de la deuxième génération et des suivantes. Ces deux initiatives se nourrissent de l'expérience d'exclusion des participants, à cause de leur apparence physique. Expérience à laquelle elles essaient de donner sens en proposant aux participants de se sentir fiers d'être des "messagers de la civilisation chinoise". Nous l'avons vu, aucune ne produit vraiment les effets escomptés par le gouvernement de la RPC. Toutefois, en consolidant les liens des participants avec la Chine, elles contribuent à la construction nationale à distance d'une autre façon. Les colonies de vacances mobilisent un réseau transnational dense de jeunes de la diaspora chinoise et les concours de beauté contribuent à entretenir l'héritage chinois. En visant les jeunes de la deuxième génération, qui construisent tous une identité hybride, la cible est bien choisie car ce sont des identités instables et constamment disputées.

Texte traduit de l'anglais par Catherine Guilyardi. 